\title{
Brachytherapy structural shielding calculations using Monte Carlo generated, monoenergetic data
}

$5 \quad$ K. Zourari, V. Peppa

Medical Physics Laboratory, Medical School, University of Athens, 75 Mikras Asias, 11527, Athens, Greece

Facundo Ballester

10 Department of Atomic, Molecular and Nuclear Physics, University of Valencia, Burjassot 46100, Spain

Frank-André Siebert

Clinic of Radiotherapy, University Hospital of Schleswig-Holstein, Campus Kiel24105, Germany

15 P. Papagiannis ${ }^{\text {a) }}$

Medical Physics Laboratory, Medical School, University of Athens, 75 Mikras Asias, 11527, Athens, Greece

20 a) Author to whom correspondence should be addressed. Electronic mail: ppapagi@phys.uoa.gr 


\begin{abstract}
Purpose: To provide a method for calculating the transmission of any broad photon

25 beam with a known energy spectrum in the range of $20 \mathrm{keV}-1090 \mathrm{keV}$, through concrete and lead, based on the superposition of corresponding monoenergetic data obtained from Monte Carlo simulation.
\end{abstract}

Methods: MCNP5 was used to calculate broad photon beam transmission data through varying thickness of lead and concrete, for monoenergetic point sources of energy in the range pertinent to brachytherapy (20 to $1090 \mathrm{keV}$, in $10 \mathrm{keV}$ intervals). The three parameter empirical model introduced by Archer et al. ${ }^{1}$ was used to describe the transmission curve for each of the 216 energy-material combinations. These three parameters, and hence the transmission curve, for any polyenergetic spectrum can then be obtained by superposition along the lines of Kharrati et al. ${ }^{2} \mathrm{~A}$

35 simple program, incorporating a graphical user interface, was developed to facilitate the superposition of monoenergetic data, the graphical and tabular display of broad photon beam transmission curves, and the calculation of material thickness required for a given transmission from these curves.

Results: Polyenergetic broad photon beam transmission curves of this work, 40 calculated from the superposition of monoenergetic data, are compared to corresponding results in the literature. A good agreement is observed with results in the literature obtained from Monte Carlo simulations for the photon spectra emitted from bare point sources of various radionuclides. Differences are observed with corresponding results in the literature for x-ray spectra at various tube potentials, 45 mainly due to the different broad beam conditions or x-ray spectra assumed.

Conclusions: The data of this work allow for the accurate calculation of structural shielding thickness, taking into account the oblique incidence of radiation to the shield, spectral variation with shield thickness, and broad beam conditions, in a 
realistic geometry. The simplicity of calculations obviates the need for the use of

50 crude transmission data estimates such as the HVL and TVL indices. Although this study was primarily designed for brachytherapy, results might also be useful for radiology and nuclear medicine facility design, provided broad beam conditions apply.

55 Key words: brachytherapy, radiation protection, shielding, Monte Carlo 
Brachytherapy structural shielding calculations using Monte Carlo generated monoenergetic data

\section{INTRODUCTION}

Broad beam radiation transmission data are essential for the structural shielding design of medical radiation facilities. Seeking to address the limited availability and disparity amongst such data for brachytherapy, a previous study presented Monte Carlo (MC) calculated transmission curves for the photon emissions of bare, point sources of radionuclides used in brachytherapy through various materials. ${ }^{3}$ A three parameter analytical representation introduced by Archer et al. ${ }^{1}$ was fit to the discrete values of transmission versus material thickness for each radionuclide-material combination. This fitting procedure was performed to obviate the need for interpolation or the use of transmission curve indices such as the half and tenth value layer (HVL and TVL, respectively). HVL and TVL do not remain constant due to spectral variation with increasing shielding barrier thickness ${ }^{3-6}$ and their use was shown to introduce potentially significant errors. ${ }^{6,7}$

70 A method for calculating the transmission of any broad photon beam in the energy range of diagnostic x-ray applications and external beam radiation therapy, using superposition of corresponding monoenergetic data in the form of the model introduced by Archer et al., ${ }^{1}$ has been presented by Kharrati et al. ${ }^{2,8}$ and Karoui and Kharrati ${ }^{9}$ As intermediate energy radionuclides are proposed for use in brachytherapy

75 to reduce shielding requirements ${ }^{3,10}$ and the use of high energy radionuclides is revived based on economic aspects, ${ }^{11}$ this work aims at extending the method of Kharrati et al. $^{2}$ to cover the energy range pertinent to brachytherapy (20 keV-1090 $\mathrm{keV}$ ) for concrete and lead, and providing a simple program to facilitate calculations. While this study was primarily designed for brachytherapy, results might also be of 80 use for radiology and nuclear medicine facility design, provided the assumed broad beam conditions apply. 
Brachytherapy structural shielding calculations using Monte Carlo generated monoenergetic data

\section{MATERIALS AND METHODS}

\section{A. Monte Carlo Simulations}

The MCNP5 v.1.40 general-purpose $\operatorname{code}^{12}$ was used for simulations of this work, with the MCNPLIB04 cross section data library which comprises a collection of data from the ENDF/B-VI.8 data library, ${ }^{13}$ based on EPDL97. ${ }^{14}$

The simulation geometry was identical to that of a previous study ${ }^{7}$ to preclude comparison bias of transmission results for brachytherapy sources. In brief, it comprised an air filled box $\left(x \times y \times z=5 \times 5 \times 3 \mathrm{~m}^{3}\right)$ with a point monoenergetic photon source centred on the $\mathrm{yz}$ plane at $\mathrm{x}=0$ and a shield barrier of thickness $\Delta \mathrm{x}$ positioned with its distal surface to the source at $\mathrm{x}=1 \mathrm{~m}$. Photons were emitted isotropically in a $2 \pi$ solid angle towards the barrier to simulate realistic broad beam conditions. The energy of the source was varied from $20-1090 \mathrm{keV}$ in $10 \mathrm{keV}$ intervals. The shield materials considered included concrete $\left(2.3 \mathrm{gcm}^{-3}\right.$ density, elemental composition taken from Hubbell and Seltzer $\left.{ }^{15}\right)$ and lead (11.35 $\mathrm{gcm}^{-3}$ density).

Radiation transmission results were calculated by the ratio of air kerma per initial photon scored for a barrier thickness $\Delta \mathrm{x}$, to that scored at the same point without any shielding barrier $(\Delta \mathrm{x}=0)$. Air kerma was scored in a spherical voxel of $20 \mathrm{~cm}$ diameter centered at $\mathrm{x}=1.3 \mathrm{~m}$ along the vertical direction from the source to the shield, using an F6 photon energy deposition tally. The evaluation of radiation transmission at a distance of $30 \mathrm{~cm}$ behind a barrier is a conservative, yet common practice in radiation shielding calculations. ${ }^{4,16}$

The resolution of barrier thickness in the set of simulations for each point source energy-shield material combination was adjusted so that three values of transmission were calculated per order of magnitude of transmission reduction, down to $10^{-4}$. The number simulated initial photon histories varied so that type A uncertainty of air kerma results expressed as the fractional error was less than $10 \%$ (for low energy 
photons at large attenuation). A rough estimate of type B uncertainty should combine that of photon interaction cross section data for the shielding materials $(2 \%$ at maximum due to uncertainty in the photoionisation cross sections ${ }^{14}$ ) and air photon mass energy-absorption coefficients (on the order of $2.3 \%$ standard uncertainty ${ }^{17}$ ).

\section{B. Broad photon beam transmission calculations from monoenergetic data}

115 The three-parameter model introduced by Archer et al. ${ }^{1}$ to facilitate diagnostic xray shielding calculations was used to describe MC calculated transmission results of this study. The model has the form:

$$
T(x, E)=\left[(1+\beta / \alpha) e^{\alpha \gamma x}-\beta / \alpha\right]^{-1 / \gamma}
$$

where $T$ stands for transmission of a broad, monoenergetic, photon beam though a shielding barrier, $x$ is the barrier thickness, $\mathrm{E}$ is the photon beam energy, and $(\alpha, \beta, \gamma)$ are constant terms to be determined by the fit that depend on photon energy and barrier material.

A weighted fit was performed using a trust region, non linear least squares algorithm, with weights inversely proportional to the square of transmission standard 125 uncertainties calculated by propagation of MC type A uncertainties.

Given $(\alpha, \beta, \gamma)$ for a material and a specific beam quality, the thickness of material required to achieve a given transmission can be calculated by solving Eq. (1) for $x$ :

$$
x=\frac{1}{\alpha \gamma} \ln \left[\frac{T^{-\gamma}+\beta / \alpha}{1+\beta / \alpha}\right]
$$

In order to obtain the transmission curve for any photon source emitting a 
transmission data results of this work $T(x, E)$ in the form of fitting results $(\alpha, \beta, \gamma)$, were combined according to the equation:

$$
\begin{gathered}
T\left[x,\left(E_{n}, f_{n}\right)\right]=\frac{\sum_{n} E_{n} f_{n}\left(\mu_{e n} / \rho\right) E_{n} T\left(x, E_{n}\right)}{\sum_{n} E_{n} f_{n}\left(\mu_{e n} / \rho\right) E_{n}}= \\
\frac{\sum_{n} E_{n} f_{n}\left(\mu_{e n} / \rho\right) E_{n}\left[\left(1+\beta_{n} / \alpha_{n}\right) \exp \left(\alpha_{n} \gamma_{n} x\right)-\beta_{n} / \alpha_{n}\right]}{\sum_{n}^{-1 / \gamma_{n}} f_{n}\left(\mu_{e n} / \rho\right) E_{n}}
\end{gathered}
$$

where $\left(\mu_{e n} / \rho\right) E_{n}$ is the mass energy absorption coefficient of air for energy $E_{n}$,

140 calculated by interpolation through $\mu_{e n} / \rho$ data available as a function of energy. ${ }^{15}$ The constants $\left(\alpha_{n}, \beta_{n}, \gamma_{n}\right)$ are also determined by interpolation through $(\alpha, \beta, \gamma)$ results of this work from fitting Eq. (1) to the MC calculated transmission of broad monoenergetic photon beams through concrete and lead.

A simple program was prepared to facilitate the calculation of transmission curves

145 through concrete and lead for monoenergetic and polyenergetic photon sources using Eq. (3). ${ }^{18}$ The graphical user interface of the program is depicted in Fig. 1. Through this interface the user can input a single photon energy or import a photon spectrum from file, provided these lie in the energy range of 20 to $1090 \mathrm{keV}$. Following selection of the shielding material (concrete or lead) from a pull-down menu, the user is presented with the options to review the transmission curve graphically, export numerical data to a file, calculate thickness required for a given transmission and tabulate results. It should be noted that since Eq. (3) cannot be readily solved for thickness, the program calculates the shielding thickness required to achieve a given transmission by interpolation though transmission curve data of resolution sufficient 155 for accurate results. 


\section{RESULTS AND DISCUSSION}

\section{A. Broad monoenergetic photon beam transmission data and fit results}

Figure 2 summarizes the results of fitting Eq. 1 to the MC generated data sets of

160 broad monoenergetic photon beam transmission, in the form of the three fitting parameters $(\alpha, \beta$, and $\gamma)$ plotted versus photon energy for concrete and lead.

According to Eq. 1, transmission tends to $\exp (-\alpha x)$ at large thickness, $x$, and $\alpha$ is the slope of a transmission curve beyond the depth where the relative directional and energy photon distributions are almost independent of thickness. Hence $\alpha$ is the

165 equivalent of an effective attenuation coefficient for the equilibrium photon spectrum at large attenuation. Its values can be seen to follow the trend of the linear attenuation coefficients for concrete and lead ${ }^{19}$ that are also plotted for comparison in Figs. 2a and $2 \mathrm{c}$, respectively, and decrease with increasing photon energy for the same material, or decreasing attenuating potential of the barrier material for the same energy. Values of

$170 \alpha$ are however lower than corresponding linear attenuation coefficients due to the build up of scattered photons. This effect is more pronounced as the relative importance of Compton interaction increases (i.e. energies greater than $40 \mathrm{keV}$ for concrete, Fig. 2a).

$\beta$ is associated to the contribution of photon build up to the broad beam

175 transmission, and its absolute value is expected to decrease as the relative importance of build up increases, as seen in Figs. $2 b$ and $2 d$ with the increase of energy for concrete and lead, respectively. $\gamma$ serves to describe the change of slope of a transmission curve at small $x$ where the equilibrium photon spectrum has not been attained, and its values can be seen to decrease with increasing energy in Figs. $2 b$ and $1802 \mathrm{~d}$, exhibiting however considerable variation. 
Corresponding results of fitting Eq. 1 to MC generated data sets of broad monoenergetic photon beam transmission have been presented in the literature in the energy range of diagnostic x-ray applications $(15 \mathrm{keV}-150 \mathrm{keV})^{8}$ and external beam radiation therapy. ${ }^{9}$ These data are also presented in Fig. 2 for comparison. Regarding $185 \alpha$ values, in Fig. 2a for concrete it can be seen that results of Kharrati et al. ${ }^{8}$ are lower than results of this work $(20 \%$ at $40 \mathrm{keV}$ and $12 \%$ at $100 \mathrm{keV})$. This is mainly due to differences in the broad beam conditions assumed since Kharrati et al. ${ }^{8}$ simulated a beam of $30 \times 30 \mathrm{~cm}^{2}$ at the shielding barrier as opposed to emission in a $2 \pi$ solid angle towards a $5 \times 3 \mathrm{~m}^{2}$ barrier simulated in this work. Hence the effect of photon 190 build up, or equivalently the departure from narrow beam conditions, represented by the linear attenuation coefficient in Fig. 2a, is greater for results of this work. On the contrary, results of Karoui and Kharrati ${ }^{9}$ are in close agreement with results of this work since they correspond to comparable broad beam conditions.

For lead, broad beam conditions and photon build up is less important relative to concrete. Hence, in Fig. $2 \mathrm{c}$ it can be seen that $\alpha$ values from the literature, ${ }^{8,9}$ are generally in excellent agreement with results of this work (within $3 \%$ ). The only exception is results of Kharrati et al. ${ }^{8}$ that exhibit an unexpected decrease in the energy range immediately after the K-edge for lead (90 keV-110 keV) and even attain negative values at $100 \mathrm{keV}$ and $105 \mathrm{keV}$ (not shown in the log scale of Fig. 2c).

200 Regarding $\beta$ and $\gamma$ values, differences between results of this work and Kharrati et $a l .{ }^{8}$ that are systematic in Fig. $2 \mathrm{~b}$ for concrete, are due to the broad beam conditions assumed in the two studies, as discussed above.

Besides graphic presentation, fitting results of this work are also provided as a table of the three fitting parameters $(\alpha, \beta$, and $\gamma)$ versus photon energy and shielding 205 material. ${ }^{20}$ This table also includes maximum differences of shielding material thickness between fit results and MC data, for the same transmission. While goodness 
Brachytherapy structural shielding calculations using Monte Carlo generated monoenergetic data

of fit metrics were excellent for all energies, these maximum differences are considerable and while not systematic and generally under than 5\%, they reach up to $14.9 \%$ for concrete and $770 \mathrm{keV}$ and $-19.6 \%$ for lead and $90 \mathrm{keV}$. They occur

210 however at large attenuation, owing to the relatively increased statistical uncertainty of results, and they are therefore considered acceptable within the scope of this work.

These findings are also presented in the comparison of $\mathrm{MC}$ data and fit results for indicative energies in Fig. 3. In this figure the greatest difference between fit results and MC data is observed for the energy of $100 \mathrm{keV}$ in lead (Fig. 3c). This is probably

215 due to the energy degradation of the equilibrium spectrum at large attenuation below the K-edge for lead, which results to an increase of transmission that the fitting equation cannot account for.

Fit results of Kharrati et al. ${ }^{8}$ are also plotted in Figs. 3a and 3c for comparison and noticeable differences from fit results of this work occur for concrete (Fig. 3a) as expected form the difference in broad beam conditions assumed in the two studies discussed above.

\section{B. Broad photon beam transmission calculations for polyenergetic brachytherapy sources}

225 Broad photon beam transmission data for polyenergetic sources used in brachytherapy can be calculated according to Eq. (3), using the fit results for monoenergetic transmission data discussed in the previous section and the program developed in this work for this task.

Indicative results of this work for ${ }^{169} \mathrm{Yb}$ and ${ }^{192} \mathrm{Ir}$ are presented in Fig. 4.

230 Corresponding MC and fit results from a previous work, ${ }^{7}$ calculated for the full spectra of photon emissions from bare, point sources of these radionuclides in the same geometry, are also presented in Fig. 4. Comparison shows excellent agreement 
for both radionuclides and structural shielding materials for transmission down to $10^{-}$

${ }^{3}$. At large attenuation, transmission differences between results of this work and MC

235 data for the full spectra are observed reaching up to $50 \%$. These differences however translate to small differences in shielding material thickness required to achieve the same transmission (i.e. $1.22 \mathrm{~cm}$ at $42 \mathrm{~cm}$ and $0.73 \mathrm{~cm}$ at $60 \mathrm{~cm}$ for ${ }^{169} \mathrm{Yb}$ and ${ }^{192} \mathrm{Ir}$ in concrete, respectively, and $-0.32 \mathrm{~cm}$ at $5.6 \mathrm{~cm}$ and $-0.07 \mathrm{~cm}$ at $1.8 \mathrm{~cm}$ for ${ }^{169} \mathrm{Yb}$ and ${ }^{192} \mathrm{Ir}$ in lead, respectively) and can therefore be considered tolerable for shielding 240 design purposes.

\section{Broad photon beam transmission calculations in the energy range of radiology and nuclear medicine applications}

The fit results for monoenergetic transmission data and the accompanying 245 program developed in this work, were also used to calculate broad photon beam transmission data through concrete and lead for x-ray spectra produced by a Tungsten anode tube of $2.5 \mathrm{~mm} \mathrm{Al}$ added filtration, operated at peak potentials of 70, 100 and $120 \mathrm{kV}_{\mathrm{p} .}{ }^{21}$ Results are presented in Fig. 5 and compared to corresponding results calculated for the same spectra using fit results of Kharrati et al., ${ }^{8}$ as well as 250 corresponding results calculated using the fit coefficients provided as a function of $\mathrm{kV}_{\mathrm{p}}$ for Tungsten anode tubes in NCRP $147^{22}$ that are adapted from the work of Simpkin. ${ }^{23}$

In Fig. 5a for concrete it can be seen that transmission results of this work are systematically higher than corresponding results in the literature owing to the 255 difference in broad beam conditions from results of Kharrati at al, ${ }^{8}$ and the combined effect of differences in broad beam conditions and the x-ray spectra assumed in the studies used to derive the data in Simpkin. ${ }^{23}$ 
Brachytherapy structural shielding calculations using Monte Carlo generated monoenergetic data

For lead, since the effect of broad beam dimensions is less important than for concrete (see Sec. II.A), results of this work were expected to be in close agreement with 260 corresponding results of Kharrati at $\mathrm{al}^{8}$ for the same $\mathrm{x}$-ray spectra. Nevertheless, in Fig. $5 b$ such agreement is only observed at small attenuation for the lower peak potentials of $70 \mathrm{kV}_{\mathrm{p}}$. Significant differences are also observed between results of this work and NCRP $147 .^{22}$

These findings imply that results of this work are not suitable in shielding calculations 265 for primary barriers in radiology due to the underlying broad beam assumptions. They could be used for secondary barrier calculations provided that the spectrum of scatter or leakage radiation is known.

In Fig. 6 results of this work are compared to corresponding MC data in the literature ${ }^{6}$ for $511 \mathrm{keV}$ photons that are of interest to the shielding design of positron emission 270 tomography facilities. A good agreement can be observed between the two data sets in concrete and lead with differences within $8 \%$. In the absence of transmission curve results for radionuclides used in nuclear medicine, results of this work were compared to lead HVL data in the literature ${ }^{24}$ and found in good agreement (i.e. $0.29 \mathrm{~mm}$ versus $0.23 \mathrm{~mm}$ for ${ }^{201} \mathrm{Tl}, 0.69 \mathrm{~mm}$ versus $0.66 \mathrm{~mm}$ for ${ }^{67} \mathrm{Ga}$, and $2.70 \mathrm{~mm}$ versus $3.0 \mathrm{~mm}$ for $\left.275{ }^{131} \mathrm{I}\right)$. A considerable difference was observed only for ${ }^{111} \mathrm{In}(0.34 \mathrm{~mm}$ versus $1.3 \mathrm{~mm})$ which, considering the effective energy of the ${ }^{111}$ In photon emissions, is attributed to an error in the NCRP report. ${ }^{24}$

The above results, combined with the fact that the broad beam conditions assumed in this work are comparable to those occurring in nuclear medicine applications, suggest 280 that results of this work can be used for nuclear medicine shielding calculations. Such calculations are expected to err on the safe side since patient absorption will not be taken into account. 
Brachytherapy structural shielding calculations using Monte Carlo generated monoenergetic data

\section{Conclusions}

Transmission data through concrete and lead were obtained for point, monoenergetic photon sources $(20 \mathrm{keV}-1090 \mathrm{keV})$ and broad beam conditions using Monte Carlo simulation. After fitting to a three parameter empirical model, these results can be combined to obtain the transmission curve for any polyenergetic spectrum. A simple program was developed to facilitate calculations as well as

290 provide graphical and tabular display of broad photon beam transmission curves, and allow for the calculation of material thickness required for a given transmission. Results of this work can be used for structural shielding design calculations for brachytherapy and nuclear medicine applications. While the broad beam conditions assumption underlying results of this work renders their use inappropriate for 295 radiology primary barrier shielding calculations, they could be used for secondary barrier calculations provided that the spectrum of scatter or leakage radiation is known.

\section{Acknowledgements}

300 This work was performed within the framework of the GEC-ESTRO working group BRAPHYQS (BRAchytherapy PHYsics Quality assurance System) physicist' network. BRAPHYQS members are deeply acknowledged for their contributions to the discussions on this topic. Companies supporting the meetings of BRAPHYQS are acknowledged for their financial support: Eckert\&Ziegler Bebig GmbH, GE Health,

305 Nucletron (an Elekta company) and Varian Medical Systems.

This research has been co-financed by the European Union (European Social Fund ESF) and Greek national funds through the Operational Program "Education and Lifelong Learning" of the National Strategic Reference Framework (NSRF) - 
Research Funding Program: Heracleitus II. Investing in knowledge society through 310 the European Social Fund. 


\section{References}

$315{ }^{1}$ B. J. Archer, J. I. Thornby, and S. C. Bushong, "Diagnostic x-ray shielding design based on an empirical model of photon attenuation," Health Phys. 44, 507-517 (1983).

${ }^{2}$ H. Kharrati, A. Agrebi, and M. K. Karaoui, "Monte Carlo simulation of x-ray buildup factors of lead and its applications in shielding of diagnostic x-ray facilities,"

320 Med. Phys. 34, 1398-1404 (2007).

${ }^{3}$ G. Lymperopoulou, P. Papagiannis, L. Sakelliou, E. Georgiou, C. J. Hourdakis, and D. Baltas, "Comparison of radiation shielding requirements for HDR brachytherapy using ${ }^{169}$ Yb and ${ }^{192}$ Ir sources," Med. Phys. 33, 2541-2547 (2006).

${ }^{4}$ NCRP (National Council on Radiological Protection and Measurements),

325 "Management of Radionuclide Therapy Patients," Report No. 155 (NCRP, Washington, DC, 2007).

${ }^{5}$ IAEA (International Atomic Energy Agency), "Radiation protection in the design of radiotherapy facilities safety,” Reports Series No. 47 (IAEA, Vienna, 2006).

${ }^{6}$ R. E. Wendt III, L. E. Williams, M. V. Yester, “AAPM Task Group 108: PET and 330 PET/CT Shielding Requirements,” Med Phys. 33, 4-15 (2006).

${ }^{7}$ P. Papagiannis, D. Baltas, D. Granero, J. Pérez-Calatayud, J. Gimeno, F. Ballester, and J. L. M. Venselaar, "Radiation transmission data for radionuclides and materials relevant to brachytherapy facility shielding," Med Phys. 35, 4898-906 (2008).

${ }^{8}$ H. Kharrati, A. Agrebi and M. K. Karaoui, "Monte Carlo simulation of photon 335 buildup factors for shielding materials in diagnostic x-ray facilities," Med. Phys. 39, 6014-6021 (2012).

${ }^{9}$ M. K. Karoui and H. Kharrati, "Monte Carlo simulation of photon buildup factors for shielding materials in radiotherapy x-ray facilities,” Med. Phys. 40, 073901 (2013). 
${ }^{10}$ S. A. Enger, D. R. Fisher, and R. T. Flynn, "Gadolinium-153 as a brachytherapy

340 isotope,” Phys. Med. Biol. 58, 957-964 (2013).

${ }^{11}$ M. Andrássy, Y. Niatsetski, J. Pérez-Calatayud, “Co-60 versus Ir-192 in HDR brachytherapy: Scientific and technological comparison,” Rev. Fis. Med. 13, 125-30 (2012).

${ }^{12}$ E.T. Booth, "MCNP-A General Monte Carlo N-Particle Transport Code, Version 5.” 345 LA-UR-03-1987 (2003).

${ }^{13}$ M. C. White, "Photoatomic Data Library MCPLIB04: A new photoatomic library based on data from ENDF/B-VI Release 8," Los Alamos National Laboratory, Internal Memorandum X-5: MCW-02-111 (2002).

${ }^{14}$ D. E. Cullen, J. H. Hubbell, and L. Kissel, EPDL97: The evaluated photon data 350 library '97,' UCRL-LR-50400 Vol 6 Rev 5 (Lawrence Livermore National Library, Springfield, VA, 1997).

${ }^{15}$ J. H. Hubbell and S. M. Seltzer 2004 Tables of X-Ray Mass Attenuation Coefficients and Mass Energy-Absorption Coefficients (version 1.4). [Online] Available: http://physics.nist.gov/xaamdi [Sunday, 07-Jul-2013 08:16:15 EDT]. 355 National Institute of Standards and Technology, Gaithersburg, MD.

${ }^{16}$ NCRP (National Council on Radiological Protection and Measurements), "Structural shielding design and evaluation for megavoltage $\mathrm{X}$ - and gamma-ray radiotherapy facilities," Report No. 151 (NCRP, Washington, DC, 2005).

${ }^{17}$ P. Andreo, D. T. Burns, and F. Salvat, "On the uncertainties of photon mass energy360 absorption coefficients and their ratios for radiation dosimetry,” Phys. Med. Biol. 57, 2117-2136 (2012).

${ }^{18}$ See supplementary material at [URL will be inserted by AIP] for a copy of the program for the calculation of transmission curves through concrete and lead for monoenergetic and polyenergetic photon sources. The program is also available 
Brachytherapy structural shielding calculations using Monte Carlo generated monoenergetic data

365 online through the BRAPHYQS link at the ESTRO web site: http://www.estro.org/about/governance-organisation/committees-activities/gec-estrobrachytherapy-committee-activities

${ }^{19}$ M. J. Berger, J. H. Hubbell, S. M. Seltzer, J. Chang, J. S. Coursey, R. Sukumar, D. S. Zucker, and K. Olsen, (2010), XCOM: Photon Cross Section Database 370 (version 1.5). [Online] Available: http://physics.nist.gov/xcom [Sunday, 07-Jul-2013 08:14:31 EDT]. National Institute of Standards and Technology, Gaithersburg, MD. ${ }^{20}$ See supplementary material at [URL will be inserted by AIP] for a table of $\alpha, \beta$, and $\gamma$ fit results versus photon energy, as well as maximum difference in shielding thickness between MC and fit results for the same transmission.

$375{ }^{21}$ J. T. Bushberg, J. A. Seibert, E. M. Leidholdt, and J. M. Boone, Appendix C in: The essential physics of medical imaging (Lippincott Williams and Wilkins, Philadelphia, 2002).

${ }^{22}$ NCRP (National Council on Radiation Protection and Measurements), "Structural shielding design for medical facilities," Report No. 147 (NCRP , Bethesda, MD, 380 2004).

${ }^{23}$ D. J. Simpkin, "Transmission data for shielding diagnostic x-ray facilities," Health Phys. 68, 704-709 (1995).

${ }^{24}$ NCRP (National Council on Radiation Protection and Measurements), "Sources and Magnitude of Occupational and Public Exposures from Nuclear Medicine 385 Procedures,” Reports Series No. 124 (NCRP, Bethesda, MD, 1996). 


\section{Figure Captions}

Figure 1. A depiction of the graphical user interface of the program prepared to

390 facilitate the calculation of broad beam transmission curves through concrete and lead for any polyenergetic photon beam, based on corresponding monoenergetic results of this work.

Figure 2. Results of fitting the three parameter model of Eq. 1 to Monte Carlo calculated broad beam transmission data of this work for monoenergetic photon sources. (a) parameter $\alpha$ for concrete compared to corresponding results from literature $^{8,9}$ and the linear attenuation coefficient, $\mu,{ }^{19}$ (b) parameters $\beta$ and $\gamma$ for concrete compared to corresponding results from the literature ${ }^{8,9}$, (c) parameter $\alpha$ for lead compared to corresponding results from the literature ${ }^{8,9}$ and the linear attenuation coefficient, $\mu,{ }^{19}$ (d) parameters $\beta$ and $\gamma$ for lead compared to corresponding results 400 from the literature ${ }^{8,9}$.

Figure 3. Monte Carlo calculated broad beam transmission data plotted versus concrete $(a, b)$ and lead $(c, d)$ thickness, for selected photon energies. Results of fitting Eq. 1 to the data, as well as corresponding fit results from the literature, ${ }^{8}$ are also plotted in Figs. (a) and (c) for comparison.

405 Figure 4. Broad beam transmission data through (a) concrete, and (b) lead, calculated using Eq. 3 with results of this work for the ${ }^{169} \mathrm{Yb}$ and ${ }^{192} \mathrm{Ir}$ photon spectra. Corresponding Monte Carlo and fit results from the literature ${ }^{7}$ are also presented for comparison.

Figure 5. Broad beam transmission data through (a) concrete, and (b) lead, calculated

410 using Eq. 3 with results of this work for x-ray spectra of a tube operated at three different peak potentials. ${ }^{21}$ Corresponding results for the same x-ray spectra using the same method with results from the literature, ${ }^{8}$ as well as corresponding results for the same peak potentials, ${ }^{22}$ are also presented for comparison. 
Figure 6. Broad beam transmission data through concrete and lead, calculated using

415 Eq. 3 with results of this work for $511 \mathrm{keV}$ photons. Corresponding Monte Carlo and fit results from the literature ${ }^{6}$ are also presented for comparison. 
Select data to create transmission curves

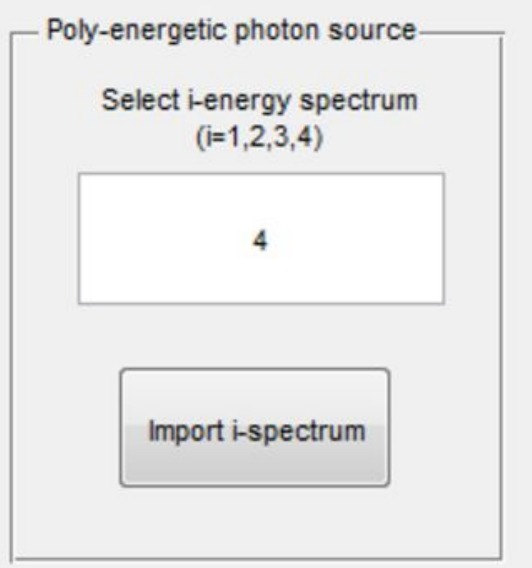

OR

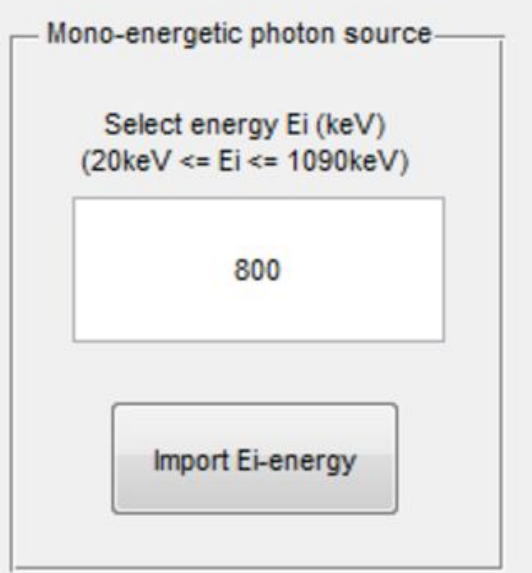

AND

Select material

$$
\text { Lead }
$$

Create Transmission Curves

\section{Concrete}

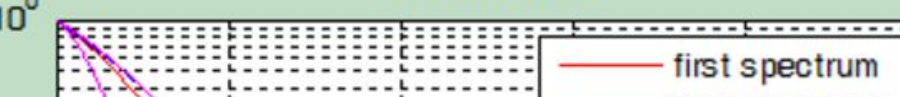
— third spectrum

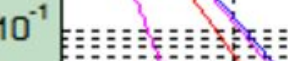
600

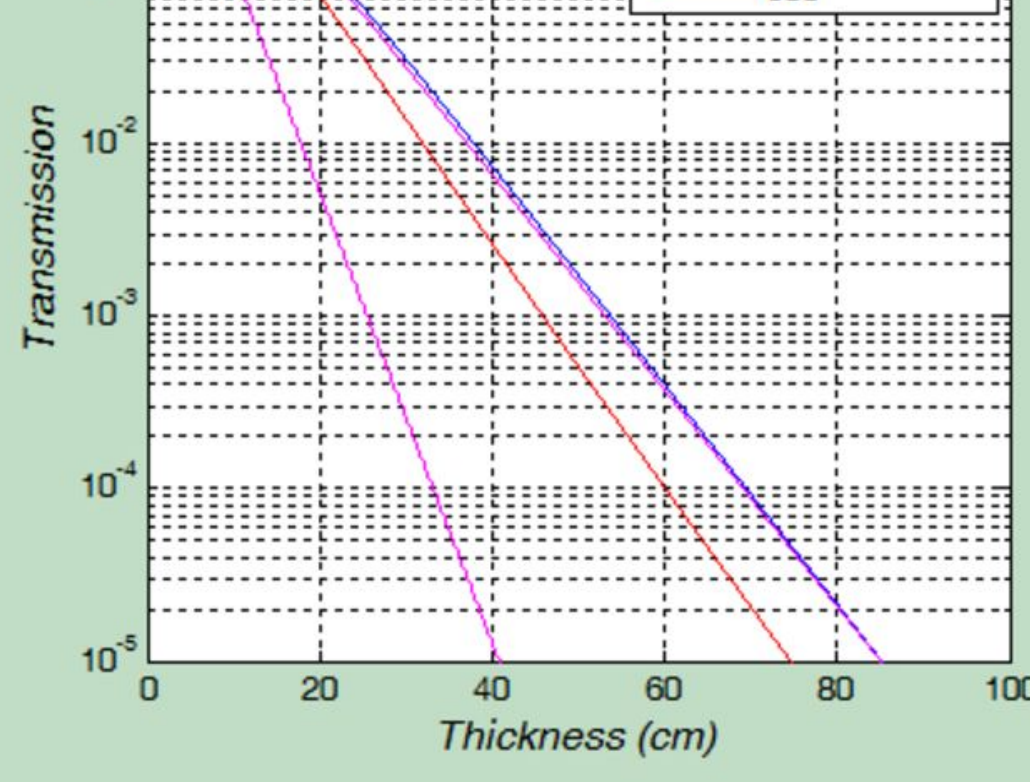

\section{Lead}

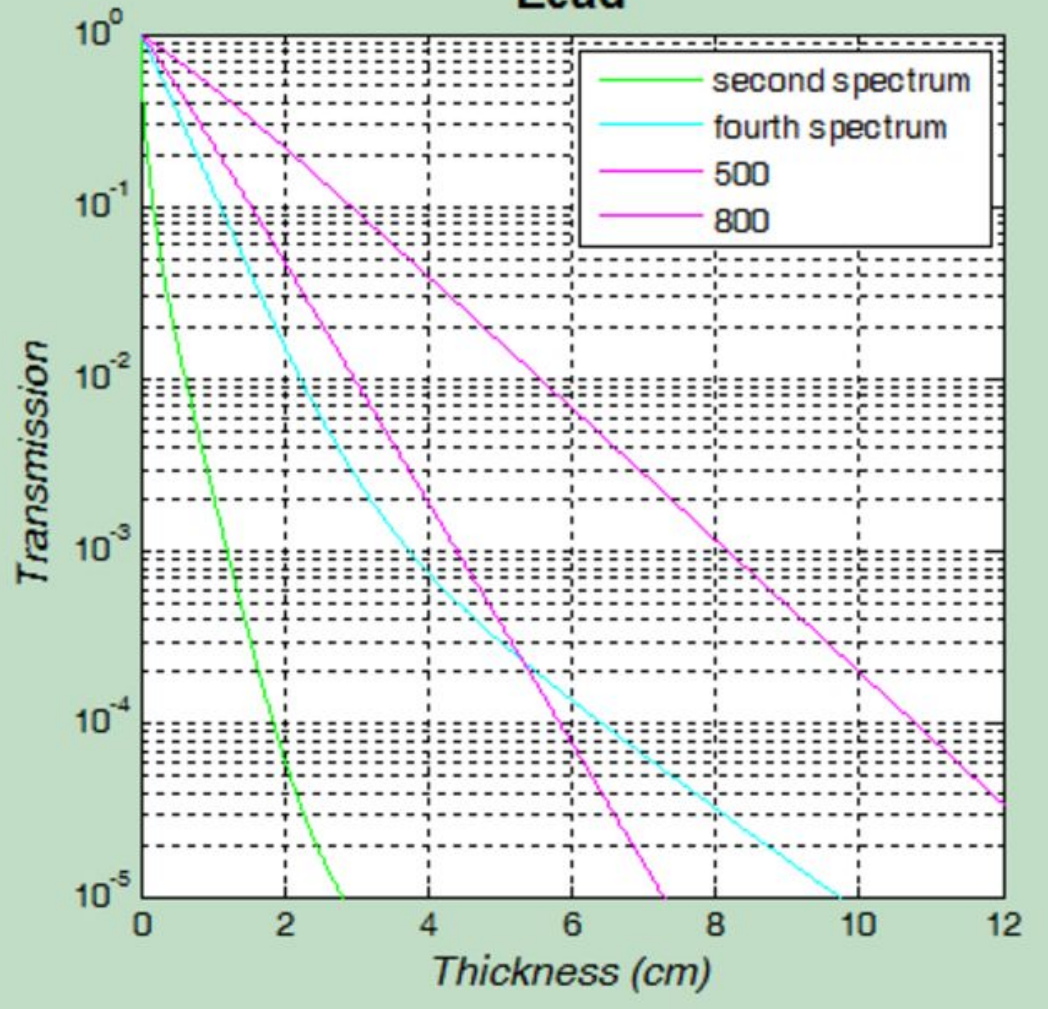

\section{- Calculate thickness for the last transmission curve-}

Transmission

0.05
Thickness (cm)

3.74209

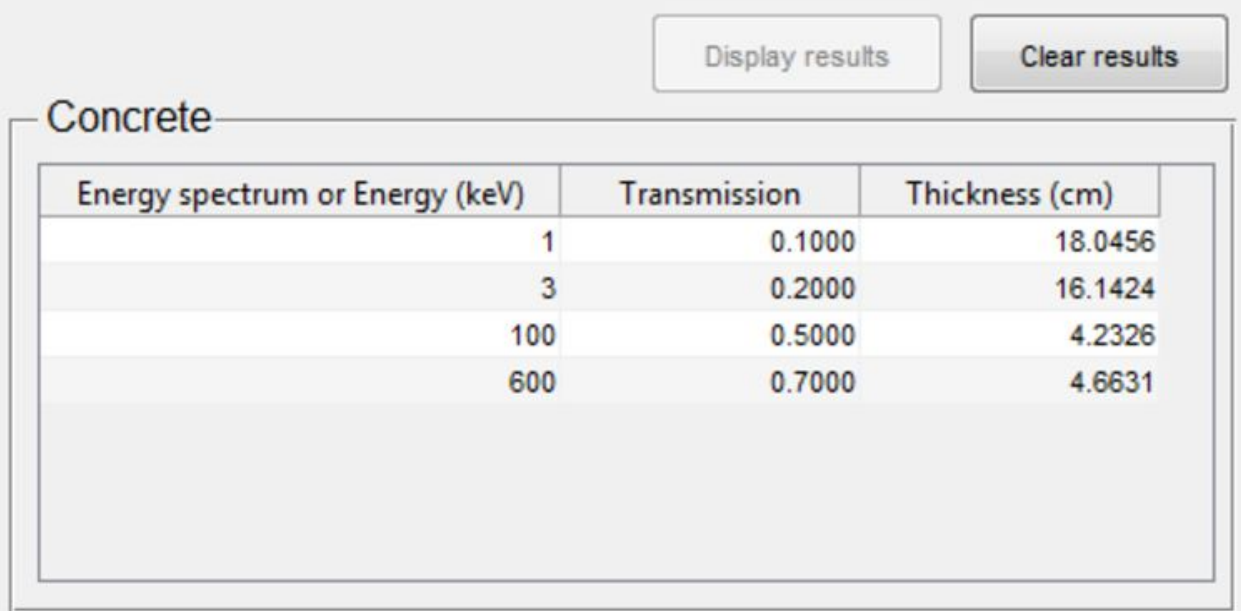

Lead-
\begin{tabular}{|r|r|r||}
\hline Energy spectrum or Energy (keV) & \multicolumn{1}{c|}{ Transmission } & Thickness (cm) \\
\hline 2 & $1.0000 \mathrm{e}-04$ & 1.8536 \\
4 & $1.0000 \mathrm{e}-05$ & 9.7244 \\
500 & 0.0030 & 3.7258 \\
800 & 0.0500 & 3.7421 \\
& & \\
\hline & & \\
\hline
\end{tabular}



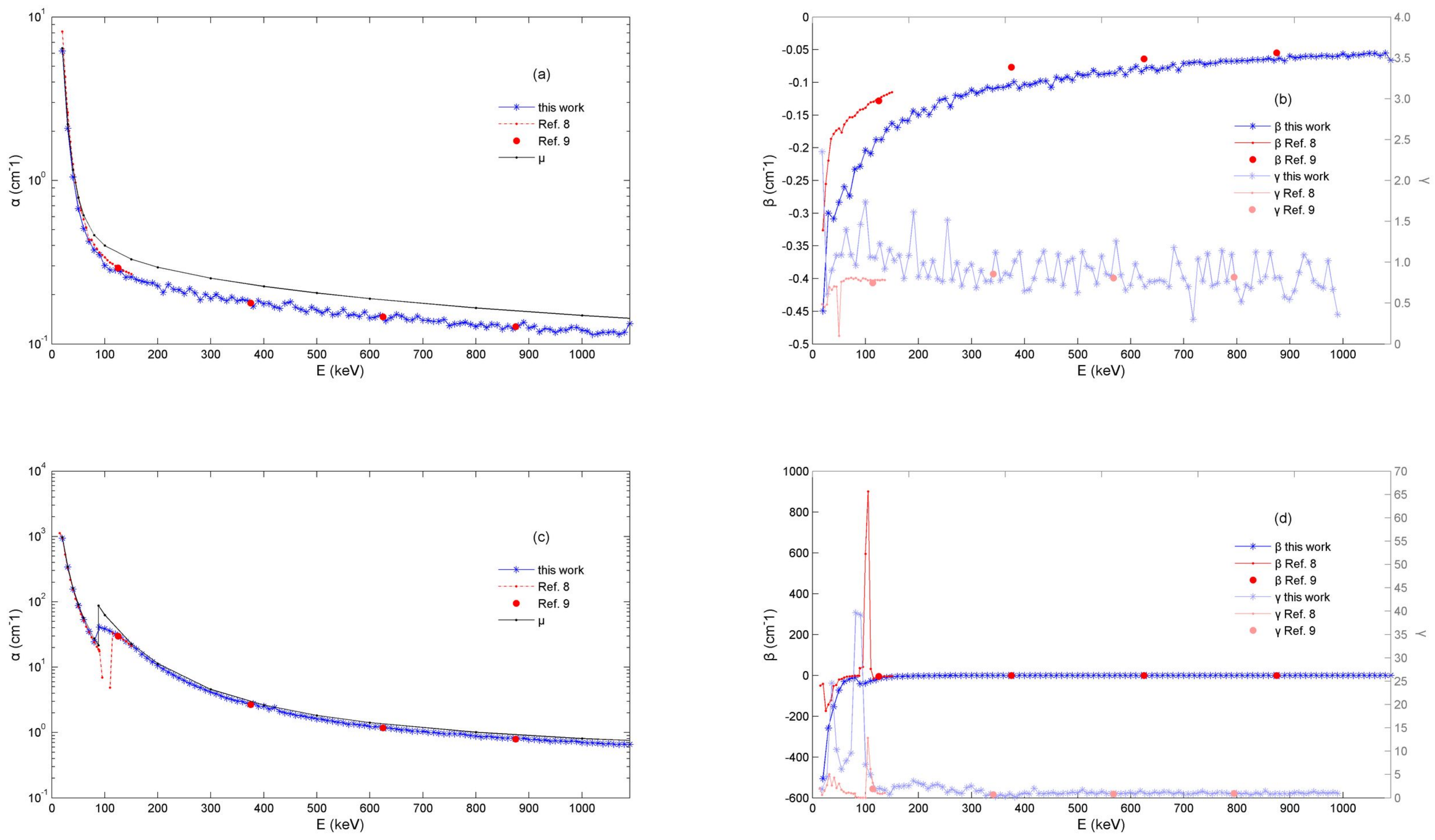

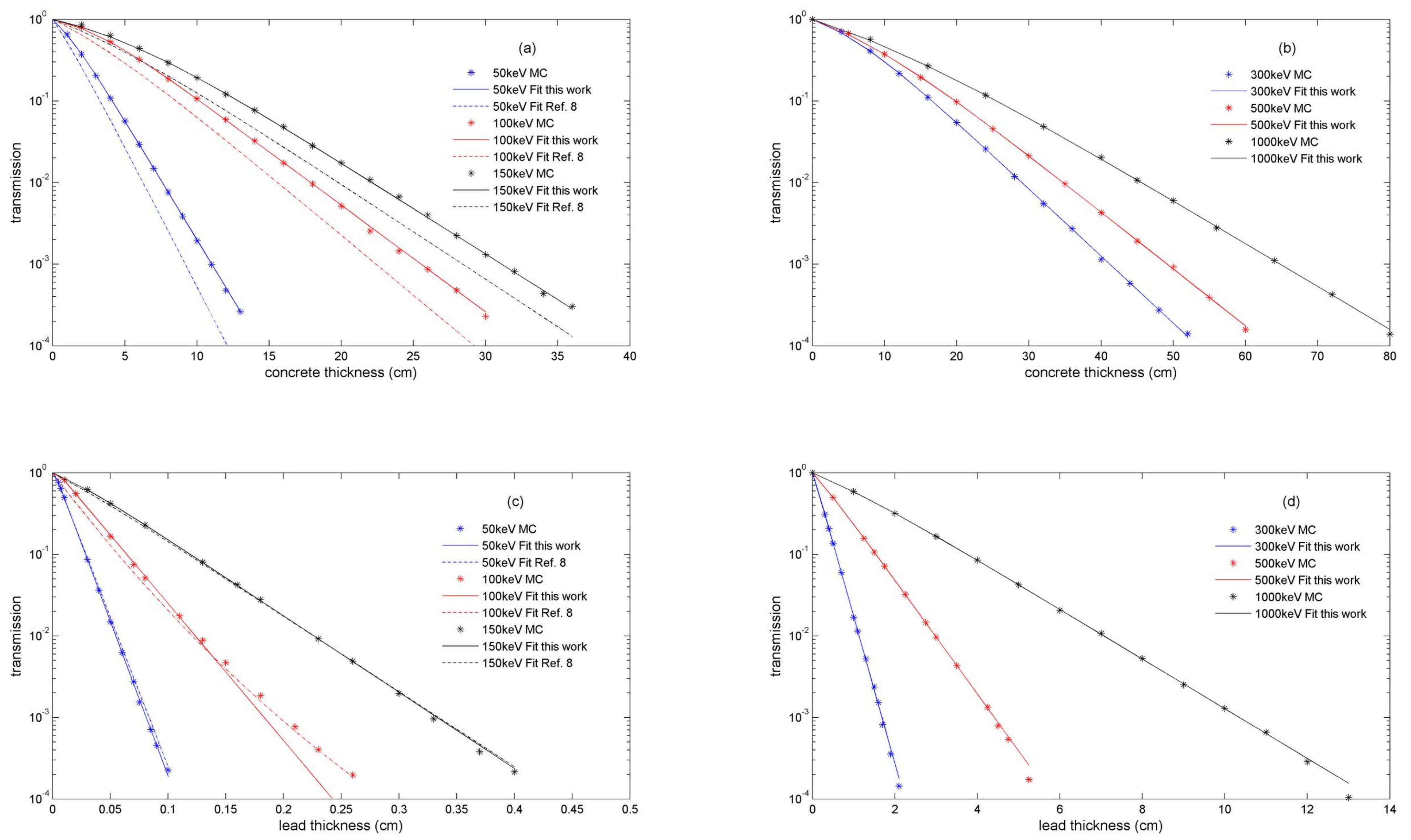

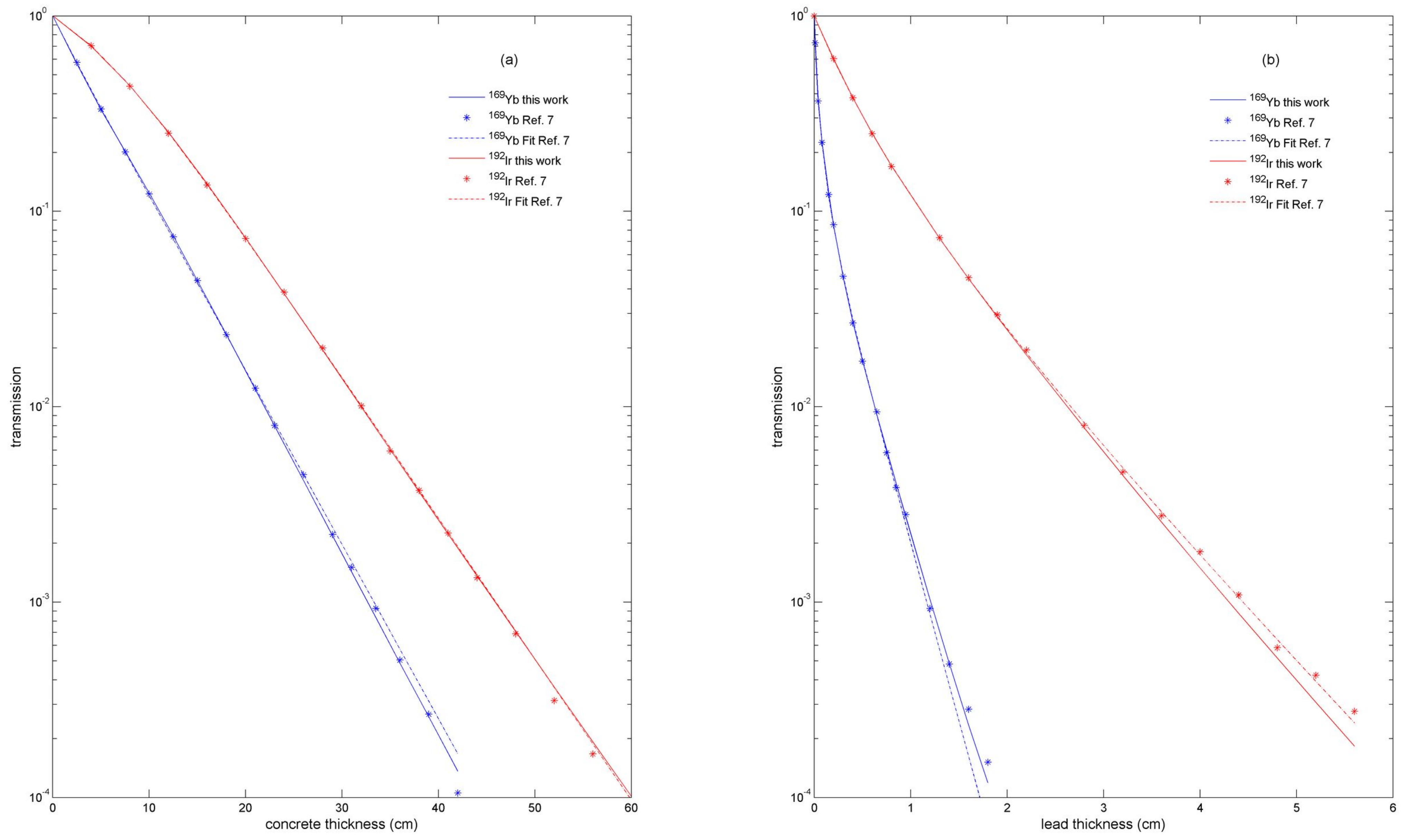

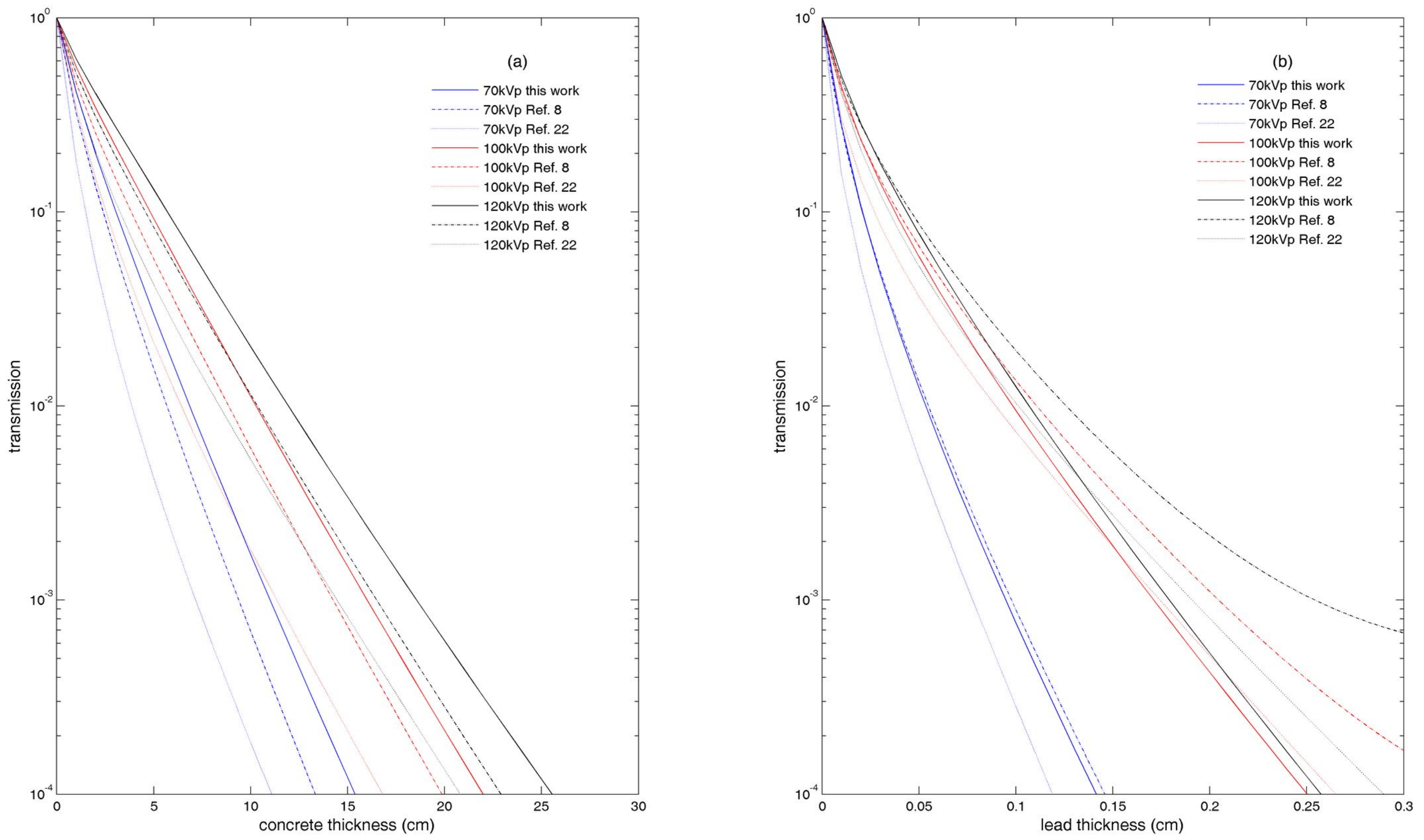


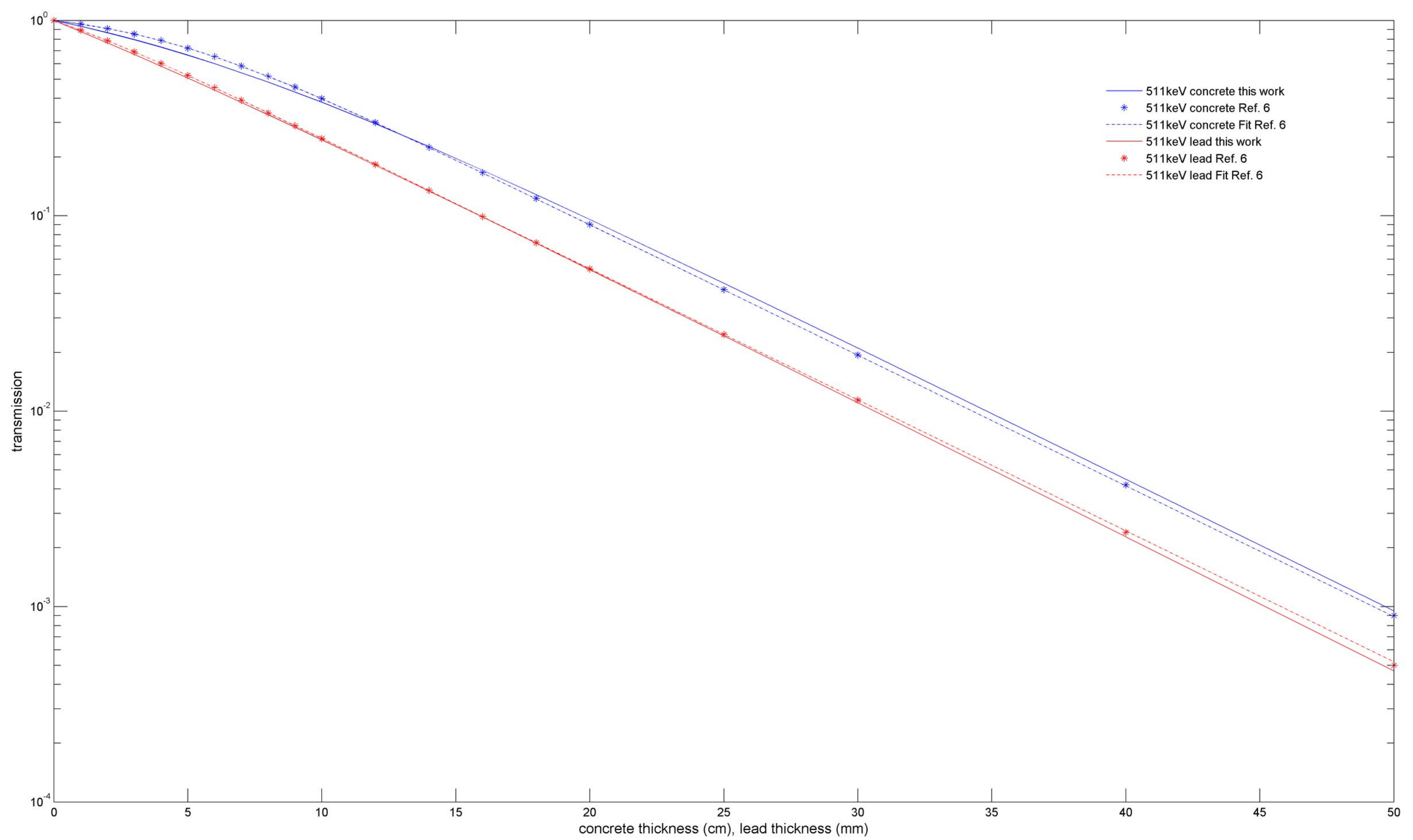

